\title{
Identification of Remarkable Dimensions in Management and Evaluation of Non-Communicable Disease Programmes A systematic review and meta-synthesis
}

\author{
Leila Vali, ${ }^{1}$ "Fatemeh Ataollahi, ${ }^{2}$ Mohammadreza Amiresmaili, ${ }^{3}$ Nouzar Nakhaee ${ }^{4}$
}

\begin{abstract}
Objectives: This study aimed to identify indicators of proper programme development in the field of non-communicable diseases through the systematic review of existing literature. Methods: In this systematic review, a search was conducted through PubMed, Scopus, EMBASE, Web of Science, Google Scholar, Cochrane Library, ProQuest and grey literature. The search was limited to literature published between January 2000 and January 2019. Results: A total of 36 articles were found relevant to the study objectives. Data were obtained through these articles based on the context, input, process and product (CIPP) model. Seven subsets for context, five subsets for input, four subsets for process and six subsets for the product were identified. Conclusion: According to CIPP subsets, indicators such as programme definition, appropriate organisational culture, structure and evaluation must be considered to develop an appropriate programme to improve health services.
\end{abstract}

Keywords: Disease Management; Program Evaluation; Health Status Indicators; Program Development.

$\mathrm{I}$ N RECENT DECADES, ONE OF THE LEADING GLOBAL challenges in the health system has been noncommunicable diseases (NCDs). ${ }^{1}$ This results in poor health, economic loss, life loss, diminished quality of life and poor social development. ${ }^{2}$ NCDs are expected to account for over $70 \%$ of all deaths globally based on the prediction of the World Health Organization (WHO). ${ }^{3}$ It is estimated that 41 million people in countries with low-resource potential will die from NCDs mainly due to cardiovascular diseases (CVDs; 48\%), cancers (21\%), chronic respiratory diseases (12\%) and diabetes (3\%). ${ }^{3}$

The WHO has predicted that appropriate interventions will prevent the spread of NCDs. ${ }^{4}$ Coping strategies for NCDs include interventions to reduce risks to the general public. These interventions change health behaviours by providing interactions among health care providers and recipients of these services through legislation, financial and tax incentives and public health promotion. ${ }^{5}$ In this regard, organisations are responsible for managing and controlling NCDs. Despite these undertakings in the field of NCDs, the incidence and prevalence of these diseases have not yet decreased significantly. ${ }^{6}$

A systematic approach to prioritising, decisionmaking and managing and improving interventions and community-based strategies is necessary to develop a programme for managing NCDs considering the limited resources available in developing countries. ${ }^{7}$ Managerial bodies in the field of NCDs have to adopt a participatory approach relying on sound scientific evidence to merge information and set long-term plans. ${ }^{8}$ Developing a proper plan depends on setting goals and priorities and the way they are shaped to draw up a clear vision of the future. To this end, development plans are based on such factors as awareness of the external environment, the parent organisation and the necessary mechanisms. ${ }^{9}$ One of the important tasks within the process of programme development is determining the success rate of the programme goal achievement. Furthermore, to influence activities and actions, developing the programme requires continuous monitoring and evaluation and optimising the movement within the designated framework. ${ }^{10}$

Dimensions of programme management and evaluation include raising community awareness, improving indicators, performing special interventions to reduce risk factors for NCDs, conducting applied research, improving cooperation and participation and establishing a system to track and evaluate progress in the field of control and prevention of NCDs. ${ }^{11}$

Evaluation helps to compare actual situations with expected outcomes of managers and policymakers towards goals and objectives. ${ }^{12}$ Different assessment patterns can be applied in the health system, including time-cost models (such as halflife chart - score model), models of organisational excellence and self-evaluation (such as Deming model, quality audit model, Malcolm Baldridge quality model and organisation excellence model) and integrated models (such as smart system, performance charter model, modelling model, goal-based management 
model, Hoshin management system model, balanced scorecard and Donabedian framework). ${ }^{13}$ The context, input, process and product (CIPP) model is a comprehensive, functional model that considers all aspects of the programme or system. ${ }^{14}$ This evaluation model is one of the most recommended frameworks for the conception, design, implementation and assessment to improve decisions emphasising constructive evaluation during the evolutionary steps of a programme. ${ }^{11}$ The four components of this model include context, input, process and product. The model contributes to the programme evaluators by collecting regular information during development and implementation. ${ }^{15}$

Despite the prevalence of NCDs and the problems caused by the chronic, progressive and debilitating nature of the diseases and various responses from the health system to focus on prevention with NCD priority, programmes in this field have not been very successful in their implementation. ${ }^{6}$ To develop a suitable programme, aspects such as accessibility, quality, efficiency and equity, that lead to the promotion of health services and a healthier community, were considered. Therefore, this study aimed to identify significant dimensions of developing programmes for the management and evaluation of NCDs through a systematic review of existing literature.

\section{Methods}

The present study is a systematic review of existing literature that aimed to identify dimensions of management and evaluation of NCDs.

Two questions were chosen for the study: 1) What remarkable dimensions should be considered for the management of programmes for NCDs?; 2) What dimensions should be considered for the evaluation of programmes for NCDs?

To identify relevant studies, selected keywords were searched in PubMed, Scopus, EMBASE, Web of Science, Google Scholar, Cochrane Library and ProQuest. Grey literature, searched through Cochrane Library and ProQuest, pertained to the period from January 2000 to January 2019. After eliminating articles that met the exclusion criteria, to increase the sensitivity and to identify all relevant studies, the references were checked, which finally led to the inclusion of three more articles. Keywords used to search the relevant literature included 'Evaluation OR assessment OR appraisal OR measurement OR audit OR accreditation', 'Developing programme', 'Noncommunicable disease OR health system, 'Framework OR model OR scheme OR pattern OR mechanism OR system,' 'Non-communicable disease OR Diabetes OR Respiratory disease OR Heart disease OR Cancer
Disease' and 'Management model OR Management pattern OR Management framework OR Management theory of diseases OR Disease management policy'.

The following inclusion criteria were used to select relevant studies: (1) Articles published between January 2000 and January 2019 in English; (2) studies that focused on the management and development of programmes in the NCD field; (3) studies related to the evaluation of the health and national plans for the evaluation and management of NCDs; and (4) studies that employed evaluating models for the health services. The following exclusion criteria were used to eliminate studies: (1) Studies published in other languages; (2) studies in the area of programme implementation; (3) performance evaluation studies unrelated to the research question; and (4) studies that were published before 2000 .

The selected keywords were fed into the database search boxes and, in some databases, the search was limited to abstracts and titles. Search results of keywords were checked by a team member for the inclusion criteria; in cases of uncertainty, decisions were made based on consensus. After identifying unrelated articles and eliminating duplicated ones, the titles of the selected articles were imported into an Excel spreadsheet. The abstracts that met the inclusion criteria and were closely related to the aims of the present study were reviewed and unrelated ones were excluded. Closely related articles were included in the final step of analysis after obtaining a minimum quality score based on the 'Strengthening the Reporting of Observational Studies in Epidemiology' (STROBE) statement. Finally, the full text of selected articles was downloaded; in case an article was not for free access, it was purchased by the Kerman University of Medical Sciences Central Library.

Qualitative evaluation, selection of related articles and data collection were performed by two researchers separately (FA and LV). Selected articles were qualitatively evaluated by researchers using the STROBE checklist. ${ }^{16}$ Any disagreement between the two evaluators was referred to a third researcher (MA). The checklist consists of 22 different sections, following which various aspects of methodology were evaluated including sampling methods, measuring variables, statistical analysis, modifiers of confounders, validity and reliability issues of the tools used and study objectives. Since not all articles exactly adhered to the Strobe checklist, scores were calculated as a percentage and the acceptance threshold was considered to be a minimum score of $65 \%$. Accordingly, studies that acquired the minimum score (65\%) from the checklist questions were included and the related data extracted to perform the meta-synthesis. 
For each study, data pertaining to the article title, first author, year of publication, place of the study and the prerequisites for programme development were extracted and manually entered into the Excel spreadsheet. Meta-synthesis was performed by collecting data from articles based on the dimensions of the CIPP model.

This study is a part of a PhD thesis in healthcare management at Kerman University of Medical Sciences that has not yet been completed. It was approved by the research ethics committee of Kerman University of Medical Sciences (Code: IR.KMU.REC.1399.272).

\section{Results}

A total of 6,633 articles were extracted during the initial search of electronic resources. Of these, 1,835 duplicated articles were removed, 4,798 relatively related articles retained and 3,035 studies excluded after evaluating the titles and abstracts; 1,763 potentially related studies remained and 706 articles were excluded after the restricting search. This restriction was done by setting a time limit, restricting keywords and searching only titles and abstracts. Subsequently, 1,057 studies were selected for text retrieval, following which 1,003 were removed; 54 studies were selected for the evaluation of the inclusion and exclusion criteria, following which 18 articles were set aside. Finally, 36 studies were selected for the analysis process [Figure 1].

Based on the above-described process, 36 articles were reviewed. Of these, five were national programmes. A total of 113 authors' participation was recorded. Five articles were from the United States, four from South Africa, three from Iran and three from Bangladesh, published across 29 journals. The first paper was published in 2005 and the latest one in 2018; while the highest frequency of publication was in 2017 and 2018 with six articles each year.

Meta-synthesis was performed according to the CIPP model. First, the content of the articles and documents was analysed and all components related to management and evaluation of NCD programmes were placed based on the relationships among the four dimensions: context, input, process and product. Finally, seven sub-categories in the context dimension, five sub-categories in the input dimension, four subcategories in the process dimension and six subcategories in the product dimension were identified.

Meta-synthesis was conducted based on the CIPP model, relying on which programme development precondition was extracted. According to the above model, prerequisites for programme development comprise four dimensions: input, context, product and process [Table 1]. Subcategories and preconditions were taken into account for these four dimensions. It is noteworthy that dimensions, subcategories and prerequisites are related to the definitions of the CIPP model. In the context dimension, the relevant elements were placed as an attempt to identify problems, needs and opportunities in the field. ${ }^{17}$ Input was evaluated to specify and assess the existing capabilities, possible programme strategies for goal achievement, intended methods for implementing strategies, organisations and other resources, including budget and time. ${ }^{18}$

Process evaluation is done to identify or predict executive problems in the field of NCDs and the desirability of the process of implementing these activities. ${ }^{18}$ In the output evaluation, the results were

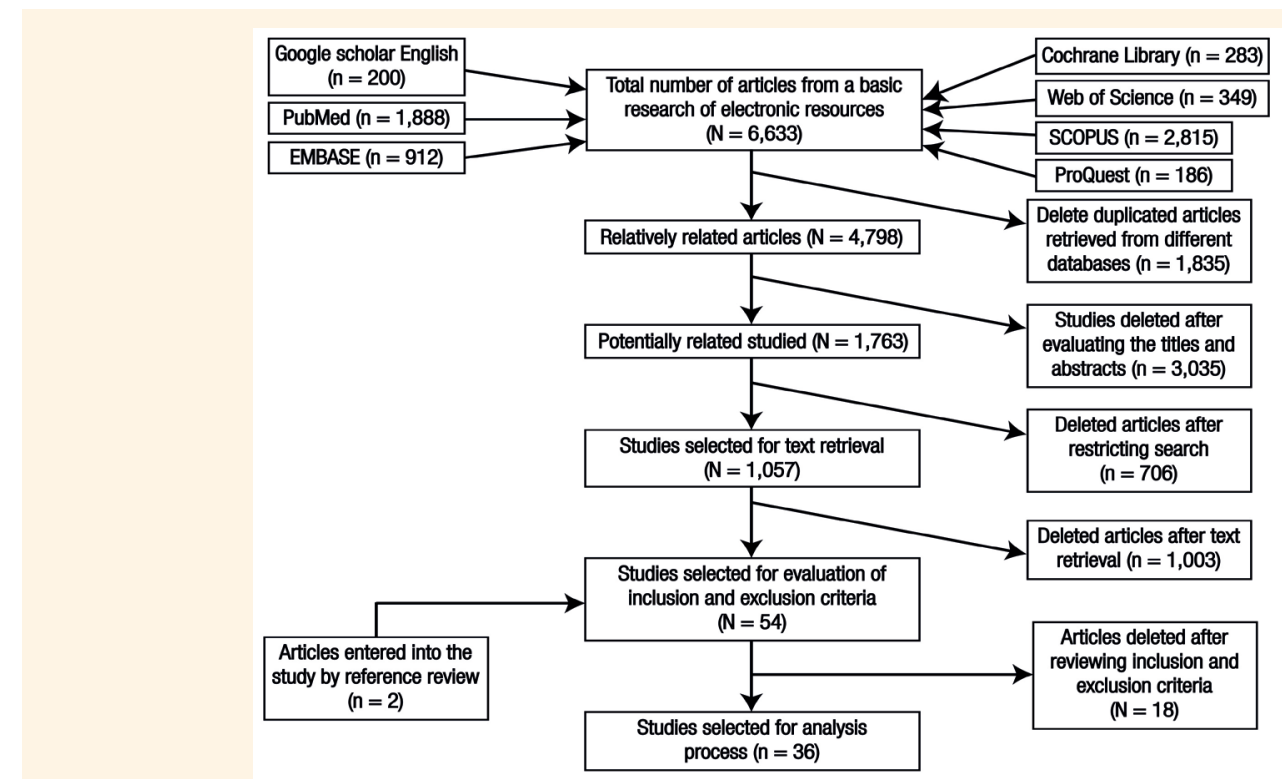

Figure 1: Flow diagram of study selection. 
Table 1: Context, input, process and product dimensions necessary for plan developing

\begin{tabular}{|c|c|c|c|}
\hline \multirow[t]{10}{*}{ Dimension } & \multirow{2}{*}{$\begin{array}{l}\text { Subset } \\
\text { Objective }\end{array}$} & Subcategory & Prerequisites \\
\hline & & Stages of development & $\begin{array}{l}\text { Considering the process of forming a team, identifying dimensions of the programme, drafting, } \\
\text { feedback to members and developing final goals }\end{array}$ \\
\hline & & Clarity & Considering realistic, clear trends in goal development \\
\hline & & $\begin{array}{l}\text { Feasibility of } \\
\text { implementation }\end{array}$ & Feasibility of implementation via considering adequate resources \\
\hline & \multirow{6}{*}{ Programme } & \multirow[t]{2}{*}{ Coverage } & $\begin{array}{l}\text { Achieving health system goals, improving community health, reducing health inequality, identifying } \\
\text { budgets and creating accountable systems }\end{array}$ \\
\hline & & & $\begin{array}{l}\text { International databases and targets, surveillance organisation, non-communicable disease control } \\
\text { and prevention centres }\end{array}$ \\
\hline & & Development & $\begin{array}{l}\text { Partnerships, clear policies, specific strategies and activities, stakeholders, outcomes, evaluation } \\
\text { criterion and resource }\end{array}$ \\
\hline & & \multirow[t]{3}{*}{ Coverage } & Developing intervention programmes \\
\hline & & & Developing an operational plan and roadmap \\
\hline & & & Developing programmes at national and international levels \\
\hline
\end{tabular}

Nature

Mission

Context
Guidelines

Executive

Input Teaching
Technology

Culture

$\begin{array}{ll} & \text { Culture } \\ \text { Organisation } & \text { Accessibility }\end{array}$

Prioritisation strategies

\section{Resources Human}

Information technology and tools

Technology in the field of people and culture and community

Motivation

(1)

Equipment

Financial

Structure Physical

Programmatic
Developing programmes at

Covering long-term, short-term and mid-term standards in the fields of home, school and workplace
health

Developing policies and documents aimed at capacity building, accessibility enhancement, cost reduction, careful monitoring and evaluation, multi-dimensionality, political commitment and prioritisation.

Developing guidelines in the areas of managerial, economic, social, legal and goal-based protocols Application of multilateral information and communication technology, management and decision support system, metadata technology and data integration and media usable technology

National and international collaborative innovative approaches

Attention to organisational incentives and creating a reward system to encourage initiatives and increase stakeholder confidence

Investigating beliefs, ethnography and identifying the impact of cultural factors on risk factors

Access to diagnostic, treatment and essential medicines

Geographical access

Access to the right budget

Participatory prioritisation considering environmental, social and cultural, legal and political contexts

Prioritisation through motivating, successful processes, stakeholder engagement and coordination Prioritisation through infrastructure identification and priority mechanisms (process, tool, method), use of evidence and public values

Developing international and national clinical guidelines to reduce disability, mortality, disease burden and appropriate design of intervention packages

Developing comprehensive interdisciplinary guidelines to implement effective, integrated, sustainable and evidence-based policies, human resources mobilisation, research, monitoring and managing complex cases

Developing comprehensive interdisciplinary guidelines to implement effective, integrated, sustainable and evidence-based policies, human resources mobilisation, research, monitoring and managing complex cases

Developing strategies to improve municipal performance (commitment to create public services for recreational spaces and parks), transportation, education (promoting healthy programmes in healthy school food distribution), tax and food industry (use of native agricultural products and natural foods)

Developing global marketing strategies, trade liberalisation, foreign investment and legal strategies to reduce demand (through price, tax)

Resource mobilisation and investment coordination to strengthen the human resources system Upgrading the capacity of specialist staff such as managers, physicians, nurses, health care staff and volunteers

Proper investment in equipment

Economic and financial tools to fund equity in sectors

Health service provider centres, provincial regional hospitals, educational consulting centres, diagnostic and pharmaceutical centres

Redesigning the system with the aim of socialising and collaborating, accessing services, supporting clinical information, self-management, managing and supporting technology and equipment and creating an organisational structure consisting of different stakeholders

Promoting capacity building in prevention and control in governmental and non-governmental service organisations

Increasing awareness through the authority delegated to local organisations to increase education and information for stakeholders and patients

National and international training programmes for professionals, students, policymakers and clients and building up of a comprehensive participatory approach to developmental aid in education and knowledge, beliefs, attitudes and skills for individuals

Developing scientific and evidence-based knowledge with the help of specialised public and private institutions to create a favourable environment for empowerment 
Table 1 (cont'd): Context, input, process and product dimensions necessary for plan developing

\begin{tabular}{|c|c|c|c|}
\hline Dimension & Subset & Subcategory & Prerequisites \\
\hline \multirow{11}{*}{ Process } & \multirow[t]{4}{*}{$\begin{array}{l}\text { Responsib- } \\
\text { ilities }\end{array}$} & & $\begin{array}{l}\text { Responsibility at the global, regional and national level for quality of service, patient safety and } \\
\text { service effectiveness }\end{array}$ \\
\hline & & & Proper accountability for noncommunicable diseases through collaboration commitment \\
\hline & & $\begin{array}{l}\text { Individuals and } \\
\text { stakeholders }\end{array}$ & $\begin{array}{l}\text { Shared responsibility and proper communication among the stakeholders to develop the plan } \\
\text { (stakeholders include community members, health professionals and non-health organizations, } \\
\text { health and non-health associations, municipalities, the private sector, religious leaders, } \\
\text { nongovernmental and local leaders and the education and training sector) }\end{array}$ \\
\hline & & $\begin{array}{l}\text { Levels of service } \\
\text { provision }\end{array}$ & Control mechanisms through communication at the service level \\
\hline & \multirow[t]{2}{*}{$\begin{array}{l}\text { Communi- } \\
\text { cations }\end{array}$} & \multirow[t]{2}{*}{$\begin{array}{l}\text { Cross-sectoral } \\
\text { communication }\end{array}$} & $\begin{array}{l}\text { Communication at different levels of society, family and individuals, agriculture, environment, } \\
\text { industry (coordination of administrative and non-administrative structure of tobacco laws, alcohol), } \\
\text { education, economy, nutrition, media, urban planning, Ministry of Information (for information), } \\
\text { collaboration between various departments of the Ministry of Health, religious organisations, trade } \\
\text { unions, the insurance industry (private and public), universities, policymakers and community } \\
\text { leaders }\end{array}$ \\
\hline & & & $\begin{array}{l}\text { Professional involvement of the national and international community in development, including } \\
\text { engagement of community, health professionals, volunteers, advisory committees and health } \\
\text { professionals }\end{array}$ \\
\hline & \multirow{4}{*}{\multicolumn{2}{|c|}{ Management }} & $\begin{array}{l}\text { Strong leadership aimed at creating comprehensive policies (national and international) and } \\
\text { monitoring activities }\end{array}$ \\
\hline & & & Clear governance and leadership in every part of the programme \\
\hline & & & $\begin{array}{l}\text { Managing and leading services such as screening, diagnosis, treatment, follow-up, case management, } \\
\text { referral, counselling, motivating, lifestyle changes, standard treatment protocol, team-based } \\
\text { care, access to core data sets, supervision, patient empowerment, social participation and policy } \\
\text { interventions }\end{array}$ \\
\hline & & & $\begin{array}{l}\text { Management in programme development to identify potential benefits, global coverage, multilateral } \\
\text { actions, definition of multi-sectoral relationships (involvement of all aspects of private / public / } \\
\text { industry / civil society / university), reduction of risk factors, coordination and cooperation }\end{array}$ \\
\hline & \multicolumn{2}{|l|}{ Leadership } & $\begin{array}{l}\text { Level of service coverage, prevention in society, the time between diagnosis and treatment, survival } \\
\text { rate and quality of life }\end{array}$ \\
\hline \multirow{17}{*}{ Product } & \multirow{2}{*}{\multicolumn{2}{|c|}{$\begin{array}{l}\text { Benefits of change in the } \\
\text { organisation }\end{array}$}} & $\begin{array}{l}\text { Use of scientific evidence and dissemination of information, goals, attention to multidisciplinary and } \\
\text { stakeholder environments, fairness of integrated actions and essential interventions }\end{array}$ \\
\hline & & & $\begin{array}{l}\text { Appropriate interventions and accountability, promoting services based on available resources and } \\
\text { infrastructure, resuscitation and reorganisation of health services, early diagnosis and integrated } \\
\text { care, documentation and monitoring of activities, multi-sectoral participatory interventions, support } \\
\text { and sensitisation of policy planners and programme managers and providers }\end{array}$ \\
\hline & \multirow{3}{*}{\multicolumn{2}{|c|}{ Health promotion }} & Development of operational plans, implementation strategies and roadmaps \\
\hline & & & Promotion of fiscal policy through tax, subsidies and system to encourage healthy nutrition \\
\hline & & & $\begin{array}{l}\text { Increasing the role of the government as a supervisor of efficient and integrated prevention and } \\
\text { control policies, allocating multilateral resources and plans and monitoring framework for plans and } \\
\text { guidelines }\end{array}$ \\
\hline & \multirow{4}{*}{\multicolumn{2}{|c|}{$\begin{array}{l}\text { Resource use effectiveness } \\
\text { assessment }\end{array}$}} & $\begin{array}{l}\text { Cost effectiveness of resources, National Health Reporting System (annually, monthly and } \\
\text { periodically) }\end{array}$ \\
\hline & & & Evaluation of programme results and programme performance based on international strategies \\
\hline & & & $\begin{array}{l}\text { Systematic evaluation and monitoring through review of the number of patients admitted, follow-up, } \\
\text { treatment (improved, died, stopped treatment, stopped follow-up) and transfer to other health care } \\
\text { centres }\end{array}$ \\
\hline & & & Documentation and policy evaluation process \\
\hline & \multirow{2}{*}{\multicolumn{2}{|c|}{ People assessment }} & Feedback of evaluation results to contacts \\
\hline & & & Assessment of trends and outcomes of interventions at the individual, social and environmental level \\
\hline & \multirow{5}{*}{\multicolumn{2}{|c|}{ Work progress assessment }} & $\begin{array}{l}\text { Evaluation and monitoring of the priorities identified and work progress, integrated disease } \\
\text { management and making the best decision }\end{array}$ \\
\hline & & & Evaluation of facilities for diagnosis and treatment \\
\hline & & & Assessment of cultural issues in prevention and control in society \\
\hline & & & Evaluation and implementation of community-based activities and community initiatives \\
\hline & & & Evaluating the impact of programmes on reducing disease risk factors \\
\hline & \multicolumn{2}{|c|}{ Information system evaluation } & $\begin{array}{l}\text { Monitoring through NCD information and data } \\
\text { Strengthen monitoring and evaluation in the area of education, research and regional strategies }\end{array}$ \\
\hline
\end{tabular}

measured against the goals of the programme and the relationship between expectations and actual results. In this study, the findings were categorised based on the definitions of the four dimensions. The context dimension showed the subsets of the context for programme development. They include the objective (stages of development, clarity, feasibility of implementation and coverage), programme (development, coverage and nature), mission, guidelines, technology, culture and organisation. The input dimension includes executive strategies, resources (human, equipment and financial), structure (physical and programmatic), teaching and learning. The process dimension includes responsibilities, communications, management and leadership. The product dimension includes the benefits of change 
in the organisation, health promotion, resource use effectiveness assessment, people assessment, work progress assessment and information system evaluation.

\section{Discussion}

This study attempted to identify crucial and effective components for management and evaluation of the programmes in the field of NCDs and the creation of a responsive system. The findings were synthesised based on the CIPP model.

In the context dimension, factors such as the objective, development and coverage programme, mission, guidelines, technology, culture and organisation were studied. In this dimension, one of the prerequisites was to consider the process of developing programme objectives appropriately as well as checking clarity, feasibility of implementation and coverage. Peykari et al. stated that clear and appropriate goals should be considered in developing national plans. Referring to a national programme for the management and control of risk factors, they suggest that developing an operational plan and roadmap, as well as providing accountability through political commitment, is one of the success factors. ${ }^{1}$ The category of technology can be considered to be an appropriate mode in the development of the programme. In this regard, Sankaranarayanan et al. have indicated that most technological responses to address personnel shortages and problems of access to health services focus on NCD programmes. For example, they demonstrated the effectiveness of using cellphone applications to check patients' compliance with medication regimens for managing diabetes and asthma. ${ }^{19}$

In the input section, some dimensions including executive strategies, resources, structure, teaching and learning have been presented. In developing NCD programmes, one of the aspects to be considered is the resource (e.g. human, equipment and financial). Nishtar suggests that a focus on human resources for health $(\mathrm{HRH})$ in the context of NCD control could have been a lever for health system change after 2015, which led to the measures designed to improve health systems more broadly. ${ }^{20} \mathrm{HRH}$ is also an important lever in the post-2015 efforts for the attainment of universal health coverage (UHC). Of all the health system resources, the most strategic ones are human, physical, technological and financial. Human resources can implement changes in the health system on a collective or individual basis. ${ }^{20}$ In programme development, providing an appropriate physical and programmatic structure is required, such as municipal commitment to creating public services, providing services centres and creating an organisational structure. In this regard, the Planning Commission of India (2003) states that, to achieve maximum impact on NCDs, programmes that are both horizontal (such as concerning public health and social welfare) and vertical (strategies focusing on populations such as controlling risk factors) should be integrated..$^{21}$

The process dimension includes responsibilities, communications, management and leadership. One of the prerequisites for programme development is that communications and multi-sectoral collaborations are taken into account. The political statement of the United Nations (2012) stated that since NCDs resulted in higher costs and lower productivity for families, individuals and societies, coordination and multi-sector communications are essential to resolve the problems through promoting tools to identify and mobilise sufficient funds. ${ }^{2}$ Moreover, the Australian National Preventive Health Agency (2010) stated that cooperation in health matters was important and one should draw a distinction between processes designed for cross-sectional coordination and the official means of increasing involvement of different sectors. ${ }^{22}$

The management of programme development includes detecting, screening and treating the diseases and providing access to palliative care for people in need. Sarink et al. concluded that coronary artery disease $(\mathrm{CAD})$ could be controlled by engaging the public in policy-making, organising, coordinating and financing. ${ }^{23}$ Public involvement can be evaluated in making policies by rules and regulation. Hence, a management framework comprising legislation, regulations and screening, as well as public education, seems necessary for preventing and controlling cardiovascular diseases. $^{23}$

The product dimension includes the benefits of change in the organisation, health promotion, resource use effectiveness assessment and assessment of people, work progress and information systems. Monitoring through the information system and data on NCDs was found important in programme development. The strategic plan of Bangladesh (2007-2010) claimed that it would emphasise NCD surveillance mechanism through the National Health Information System which generates data and publish them in database form which provides access to information for policymakers, public health managers and researchers globally. ${ }^{24}$ One of the prerequisites in the product dimension is the issue of health promotion which can be met through appropriate interventions and accountability, promoting available resources and infrastructure, integrated care, documentation and monitoring of activities and multi-sectoral support of policymakers, programme managers and providers. In a study by Diem et al., the role of capacity building 
was established through a comprehensive approach to public health interventions such as creating processes of improvement, knowledge production, increasing need evaluation, examining the characteristics of the target population and identifying the resources and required procedures. ${ }^{25}$ Mondal et al. stated that, through appropriate approaches towards interdisciplinary leadership, the shift from treatment to prevention, guideline development on interdisciplinary communication education, community access and investment within the world framework have adopted a healthier approach to accountability. ${ }^{26}$

It is worth noting that in programme development, it is crucial to have common responsibility and proper communication among the stakeholders including community members, health professionals and nonhealth organisations, municipalities, the private sector, religious leaders, nongovernmental and local leaders and the Ministry of Education. Today, global health due to the prevalence and incidence of NCDs is in a state of crisis and the health system is trying to respond to this crisis. ${ }^{2}$ Proper planning and management are essential at all levels (primary, secondary and tertiary) in all types of organisations (public, private and non-profit). Therefore, to develop, implement and evaluate the appropriate programme, a framework and a collective approach should be considered in a comprehensive view of all effective factors to promote community health.

This study systematically considered the components of appropriate programme development. Although existing literature has already considered the dimensions of developing a programme in detail, a comprehensive review did not exist. Therefore, this study improves the current knowledge of this field by collecting and categorising all necessary dimensions for developing a programme.

In this article, data were collected based on the CIPP model. It is acknowledged that other models are available for this purpose; using any other model may change the results. Moreover, limited access to the full text of some articles and documents could be the reason that some studies have not been reviewed. The fact that less robust evidence was reviewed for developed national and strategic programmes could be regarded as another limitation of the present study. However, this study offered an opportunity to study substantial plans and valuable insights.

\section{Conclusion}

Certain dimensions are essential for programme development, including engaging all key stakeholders, providing required resources and addressing cultural issues. Furthermore, a proper development of programmes should take into account such issues as developing, implementing and evaluating programmes and all components such as context, input, process and product factors. This study thereby provides valuable information for policy-makers and planners to develop appropriate programmes in a sound and cost-effective manner.

\section{AUTHORS' CONTRIBUTION}

LV and FA conceptualized the idea, while LV, FA and MA designed the study. LV, FA and NN collected the data. LV, FA and MA provided analysis and interpretation of the data. LV and FA provided the first draft of manuscript. LV, FA and MA revised the manuscript. All authors approved the final version of this manuscript.

\section{References}

1. Peykari N, Hashemi H, Dinarvand R, Haji-Aghajani M, Malekzadeh R, Sadrolsadat A, et al. National action plan for non-communicable diseases prevention and control in Iran: A response to emerging epidemic. J Diabetes Metab Disord 2017; 16:3. https://doi. org/10.1186/s40200-017-0288-4.

2. Political declaration of the high-level meeting of the General Assembly on the prevention and control of non-communicable diseases. New York: United Nations; 2011 (66/2; http://www.who. int/nmh/events/un_ncd_summit2011/en/ Accessed: Oct 2020.

3. World Health Organization. Noncommunicable Diseases (NCD). From: https://www.who.int/gho/ncd/mortality_morbidity/en/ Accessed: Oct 2020.

4. Ruby A, Knight A, Perel P, Blanchet K, Roberts B. The effectiveness of interventions for non-communicable diseases in humanitarian crises: a systematic review. PloS one 2015; 10:e0138303.

5. Mannava P, Abdullah A, James C, Dodd R, Annear PL. Health systems and noncommunicable diseases in the Asia-Pacific region: A review of the published literature. Asia Pac J Public Health 2015; 27:NP1-NP19. https://doi.org/10.1177/1010539513500336.

6. Organization WHO. Global status report on noncommunicable diseases 2014: World Health Organization; 2014.

7. Mendis S, Davis S, Norrving B. Organizational update: The World Health Organization global status report on noncommunicable diseases 2014; one more landmark step in the combat against stroke and vascular disease. Stroke 2015; 46:e121-2. https:// doi.org/10.1161/strokeaha.115.008097.

8. Geneau R, Stuckler D, Stachenko S, McKee M, Ebrahim S, Basu S, et al. Raising the priority of preventing chronic diseases: A political process. Lancet 2010; 376:1689-98. https://doi.org/10.1016/ S0140-6736(10)61414-6.

9. Doulabi RZ, Asnaashari E. Identifying success factors of healthcare facility construction projects in Iran. Procedia Eng 2016; 164:409-15. https://doi.org/10.1016/j.proeng.2016.11.638.

10. Woodward M. Epidemiology: Study Design and Data Analysis. Boca Raton: Chapman and Hall/CRC, 2013.

11. World Health Organization. Prevention and Control of Noncommunicable Diseases: Implementation of the global strategy. From: www.apps.who.int/gb/ebwha/pdf_files/EB126/ B126_12-en.pdf/ Accessed: Oct 2020. 
12. Valmohammadi $\mathrm{C}$, Ahmadi $\mathrm{M}$. The impact of knowledge management practices on organizational performance: A balanced scorecard approach. J Enterp Inf Manag 2015; 28:131-59. https://doi.org/10.1108/JEIM-09-2013-0066.

13. Anh VTK. Evaluation models in educational programme: Strengths and weaknesses. VNU J Foreign Stud 2018; 34. https://doi.org/10.25073/2525-2445/vnufs.4252.

14. Stufflebeam DL, Madaus GF, Kellaghan T. Evaluation models: Viewpoints on educational and human services evaluation. Springer Science \& Business Media, 2006.

15. Zhang G, Zeller N, Griffith R, Metcalf D, Williams J, Shea C, et al. Using the context, input, process, and product evaluation model (CIPP) as a comprehensive framework to guide the planning, implementation, and assessment of servicelearning programs. Journal of Higher Education Outreach and Engagement. 2011; 15:57-84.

16. Von Elm E, Altman DG, Egger M, Pocock SJ, Gøtzsche PC, Vandenbroucke JP, et al. The Strengthening the Reporting of Observational Studies in Epidemiology (STROBE) statement: Guidelines for reporting observational studies. Lancet 2007; 370:1453-7. https://doi.org/10.1016/S0140-6736(07)61602-X.

17. Stufflebeam, D. L. The CIPP model for evaluation. In: Stufflebeam DL, Kellaghan T, Eds., The International Handbook of Educational Evaluation. Boston: Kluwer Academic Publishers, 2003.

18. Stufflebeam DL, Shinkfield, AJ. Evaluation Theory, Models, \& Applications. San Francisco: Jossey-Bass, 2007.

19. Sankaranarayanan R, Nene BM, Shastri SS, Jayant K, Muwonge R Budukh AM, et al. HPV screening for cervical cancer in rural India. N Engl J Med 2009; 360:1385-94. https://doi.org/10.1056/ NEJMoa0808516 pmid: 19339719.
20. Nishtar S. Choked Pipes: Reforming Pakistan's health system. Oxford: Oxford University Press, 2010. https://doi.org/10.2471/ BLT.10.079285

21. Planning Commission of India. Public Health Care System (Chapter VIII), 2000. From: https://niti.gov.in/planningcommi ssion.gov.in/docs/aboutus/committee/strgrp/stgp_fmlywel/ sgfw_ch8.pdf Accessed: Oct 2020.

22. Federal Register of Legislation. Australian National Preventive Health Agency Act 2010. From: https://www.legislation.gov.au/ Details/C2016C00720 Accessed: Oct 2020.

23. Sarink D, Nedkoff L, Briffa T, Shaw JE, Magliano DJ, Stevenson C, et al. Projected age- and sex-specific prevalence of cardiovascular diseases in Western Australian adults from 2005-2045. Eur J Prev Cardiol 2016; 23:23-32. https://doi.org/10.1177/204 7487314554865.

24. Bangladesh, Ministry of Health and Family Welfare. Strategic Plan for Surveillance and Prevention of Non-Communicable Diseases in Bangladesh 2011-2015. Dhaka: Directorate General of Health Services, Ministry of Health and Family Welfare, 2007.

25. Diem G, Brownson RC, Grabauskas V, Shatchkute A, Stachenko S. Prevention and control of noncommunicable diseases through evidence-based public health: Implementing the NCD 2020 action plan. Glob Health Promot 2016; 23:5-13. https://doi. org/10.1177/1757975914567513.

26. Mondal S, Van Belle S. India's NCD strategy in the SDG era: Are there early signs of a paradigm shift? Global Health 2018; 14:1-9. https://doi.org/10.1186/s12992-018-0357-6. 